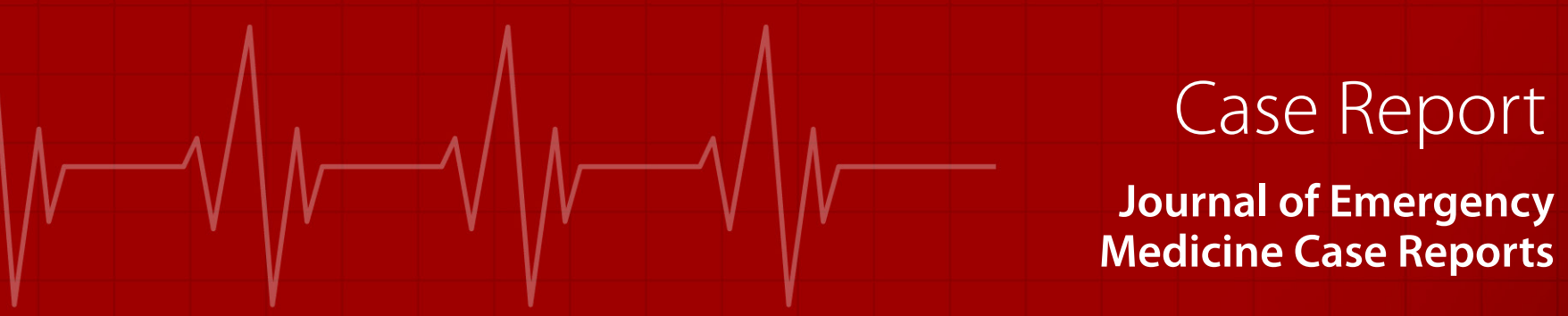

\title{
Spontaneous Spleen Rupture and Apixaban
}

Birdal Yıldırım', Ethem Acar ${ }^{1}$, Ahmet Demir ${ }^{1}$, Ahmet Aksakal' ${ }^{1}$ Kemal Gökçek', Özcan Başaran²

${ }^{1}$ Muğla Sıtkı Koçman University, Faculty of Medicine, Department of Emergency Medicine

${ }^{2}$ Muğla Sıtkı Koçman University, Faculty of Medicine, Department of Cardiology

\begin{abstract}
Novel oral anticoagulants (NOACs) are now widely used. Spontaneous splenic rupture (SLR) is a rare condition. It is life-threatening, especially if not immediately recognized. Previous studies have shown an association between NOAC use and SLR. We report a case of 53-year-old female patient who responded to cardiopulmonary resuscitation (CPR) after SLR. The patient, who had an external cardiac device for heart failure 13 months ago was admitted to our emergency department. She was unconscious, pale, hypotensive and had tachycardia. Free fluid was detected in the peritoneum by bedside ultrasonography. The patient was resuscitated for 20 minutes after arrest during the follow-up period. She was stabilized and computed tomography showed a large subcapsular splenic hematoma with active extravasation around the spleen, but persistent low urine output developed with hypotension despite resuscitation. She was taken to the operating room for splenectomy and abdominal washing. She was then recovered and discharged 17 days later. We wanted to draw attention to a patient with SLR due to NOACs. Rapid diagnosis and aggressive early resuscitation are critical in the management of NOAC associated SLR.
\end{abstract}

Keywords: Resuscitation, NOAC, spontaneous splenic rupture

\section{Introduction}

Novel oral anticoagulants (NOACs) are more preferred today. Non-traumatic, spontaneous splenic rupture is very rare and especially life-threatening if not immediately recognized ${ }^{1,2}$. Although organ-specific bleeding is much rare with NOACs compared to warfarin use, there is still a risk of intracranial bleeding and gastrointestinal (GI) bleeding ${ }^{3}$. Splenic rupture is rare compared to these bleeding complications. In many cases, splenic rupture is associated with an underlying pathological process (atraumatic-pathological rupture) rather than being atraumatic and idiopathic. Previous studies have shown an association with the use of NOACs and spontaneous splenic rupture in patients with risk factors ${ }^{4,5}$ and underlying serious chronic diseases $^{6,7}$. Although splenic rupture is well known in infectious mononucleosis, more atypical relationships with infections include follow-up of intravenous immunglobulin therapy for pure red cell aplasia associated with babesiosis ${ }^{4}$ and parvovirus $\mathrm{B} 19^{8}$. In the case of ectopic pregnancy in the spleen', the underlying pathological mechanism is more clear. Gonzva and colleagues described the first non traumatic spontaneous splenic rupture case associated with NOAC use in a 67-yearold patient from France with an acute shock presentation that was good after resuscitation, splenectomy and intensive care ${ }^{10}$. Another case was described with spontaneous splenic rupture, who was at age 57 and anticoagulated for pulmonary embolism resulted with shock ${ }^{11}$.

\section{Case Description}

We present a case of a 53-year-old female patient responding to CPR after arrest due to spontaneous splenic hemorrhage. The patient underwent an external cardiac device 13 months ago due to heart failure was admitted to our emergency department. She was unconscious, pale, hypotensive and had tachycardia. Bedside ultrasonography detected free fluid in the peritoneum. The patient was arrested during the follow-up period and was revived with 20 minutes of CPR following endotracheal intubation and underwent massive transfusion protocol. The patient was given 4 units of packed red blood cells, 1 unit of fresh frozen plasma, prothrombin complex concentrate and calcium. The first hemoglobin and hematocrit were $9.2 \mathrm{~g} / \mathrm{dL}$ per microliter and $28.4 \%$ respectively. The second hemoglobin was $6.0 \mathrm{~g} / \mathrm{dL}$ and hemotocrit was $18.4 \%$ after three hours of admission. The patient's prothrombin time and international normalized rate were 19.5 seconds and 3.89. Patient's blood pressure and heart rate improved. Computed tomography of the relatively stabilized patient (Figure 1) showed intra-peritoneal hematoma with 


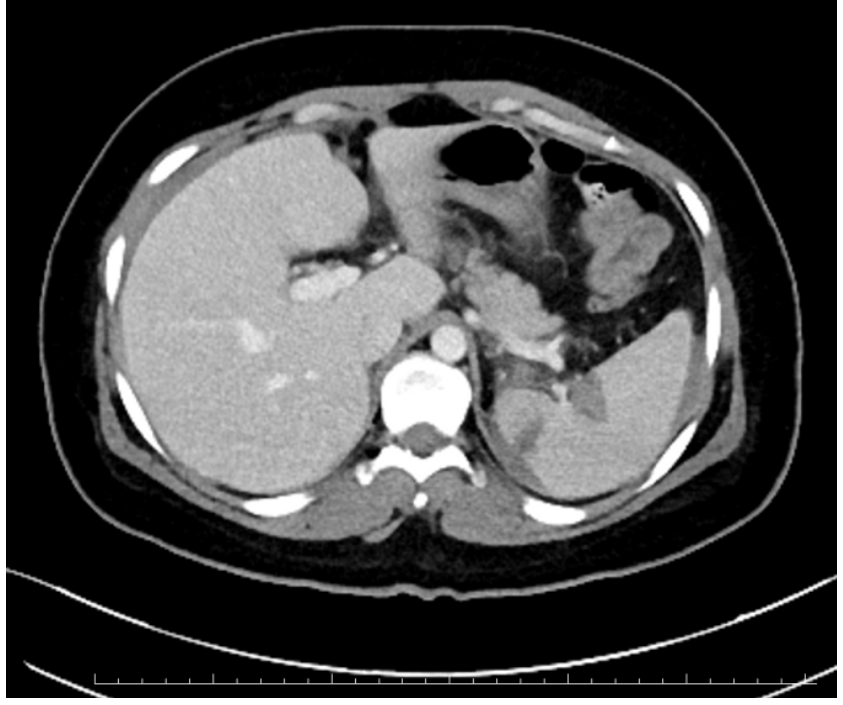

Figure 1.

active extravasation around the spleen, and despite resuscitation, persistant low urine output with hypotension developed. She was taken to the operating room for a splenectomy and abdominal wash. She recovered after the post-op intensive care unit follow-up and was discharged 17 days later. We wanted to draw attention to a case of arrest as a result of non-traumatic spontaneous splenic rupture due to NOACs. Rapid diagnosis and aggressive early resuscitation are critical in the management of spontaneous splenic rupture.

\section{Discussion}

Atraumatic-pathological splenic rupture includes neoplastic (29.3\%), infectious $(28.3 \%)$, inflammatory (20\%), iatrogenic $(10.2 \%)$ and mechanical (7.2\%.) etiology and rarely drug assocaited cases were described ${ }^{1}$. Mortality was associated with advanced age, the underlying neoplastic process, known history of splenomegaly, and especially with delay in diagnosis ${ }^{2}$. With the popularity of NOACs, as well as the evolving pharmacological strategies for bleeding control (fresh frozen plasma, activated prothrombin concentrates, recombinant activated Factor VII, idaricizumab), it is important to recognize this rare but increasingly well documented problem of atraumatic splenic rupture. Here we present a case that previously appeared relatively healthy, 53 years old, using an external heart device, with shock. We wanted to draw attention to intra abdominal bleeding suspicion with bedisde ultrasonography, rapid resuscitation with blood product transfusion and rapid surgery with treatment.

\section{Conclusion}

Spontaneous splenic hemorrhage should be bear in mind in a patient on apixaban treatment admitted with shock. Rapid diagnosis and aggressive early resuscitation resulted with full healing of our patient. Accurate diagnosis in a minimum of time was the major key in the management of this patient.

\section{Declaration of competing interest \\ None}

\section{References}

1. Khan RN, Jindal V. Systematic review of atraumatic splenic rupture. British Journal of Surgery. $\mathrm{Br} J$ Surg. 2010 Apr;97(4):616; author reply 616-7. doi: 10.1002/bjs.7052.

2. Kimura M, Hoshina K, Kobayashi M, Yamamoto S, Ohshima M, Watanabe T. Morphological analysis using geometric parameters for splenic aneurysms. Asian Cardiovasc Thorac Ann. 2018 Feb;26(2):133-138. doi: 10.1177/0218492318757040. Epub 2018 Jan 24.

3. Vanassche T, Hirsh J, Eikelboom JW, Ginsberg JS. Organ-specific bleeding patterns of anticoagulant therapy: lessons from clinical trials. Thromb Haemost. 2014;112(5):918-23.

4. Khateeb D, Santarelli R, Wexler P, Young D. Hemorrhagic Shock Due To Spontaneous Splenic Rupture: A Rare Complication Of Babesiosis. Crit Car Med. 2019;47(1):300.

5. Azar F1, Brownson E, Dechert T. Cocaine-associated hemoperitoneum following atraumatic splenic rupture: a case report and literature review. World J Emerg Surg. 2013 Aug 28;8(1):33. doi: 10.1186/1749-7922-8-33.

6. Nagaraja V, Cranney G, Kushwaha V. Hemorrhagic Shock from Spontaneous splenic rupture due to rivaroxaban. BMJ Case Rep. 2018 Mar 5;2018. pii: bcr-2017-223237. doi: 10.1136/bcr2017-223237.

7. Abdelhady A, Ahmed A, Mohamed Y, Binchy J. Apixaban-Associated Spontaneous Splenic Rupture-A Case Report. Ir Med J. 2018 Aug 13;111(7):792

8. Crabol Y, Terrier B, Rozenberg F, Pestre V, Legendre C, Hermine O, Montagnier-Petrissans C, Guillevin L, Mouthon L; Groupe d'experts de I'Assistance Publique-Hôpitaux de Paris. Intravenous immunoglobulin therapy for pure red cell aplasia related to human parvovirus b19 infection: a retrospective study of 10 patients and review of the literature. Clin Infect Dis. 2013 Apr;56(7):968-77. doi: 10.1093/cid/cis1046. Epub 2012 Dec 12.

9. Carta G1, D'Alfonso A, Nallbani A, Palermo P, Franchi V, Patacchiola F. Spontaneous rupture of splenic hemangioma in puerperium. Clin Exp Obstet Gynecol. 2012;39(3):407-8.

10. Gonzva J, Patricelli R, Lignac D. Spontaneous splenic rupture in a patient treated with rivaroxaban. Am J Emerg Med. 2014;32(950):e3.

11. Alexander Janke, Suzette Ikejiani, Charles Mize. Spontaneous splenic hemorrhage in a patient on apixiban. December 19, 2019DOI:https://doi.org/10.1016/j.ajem.2019.12.006/s 